\title{
Diet and Chemotherapy: The Effects of Fasting and Ketogenic Diet on Cancer Treatment
}

\author{
Francesco Plotti $^{a}$ Corrado Terranova ${ }^{a}$ Daniela Luvero ${ }^{a}$ Martina Bartolone ${ }^{a}$ \\ Giuseppe Messina $^{a}$ Laura Feole $^{a}$ Stefano Cianci ${ }^{b}$ Giuseppe Scaletta $^{b}$ \\ Claudia Marchetti $^{b}$ Violante Di Donato ${ }^{c}$ Anna Fagotti ${ }^{b}$ Giovanni Scambia ${ }^{b}$ \\ Pierluigi Benedetti Panicic Roberto Angioli ${ }^{\mathrm{a}}$
}

\begin{abstract}
${ }^{a}$ Department of Obstetrics and Gynaecology, Campus Bio-Medico, University of Rome, Rome, Italy; ${ }^{b}$ Gynecologic Oncology Unit, Women Wealth Area, Department of Woman and Child Health and Public Health, Fondazione Policlinico Universitario A. Gemelli IRCCS, Università Cattolica del Sacro Cuore, Rome, Italy; 'Department of Gynecological and Obstetric Sciences, and Urological Sciences, University of Rome "Sapienza", Umberto I Hospital, Rome, Italy
\end{abstract}

\author{
Keywords \\ Cancer - Chemotherapy · Diet · Fasting · Ketogenic diet . \\ Oncology $\cdot$ Treatment
}

\begin{abstract}
Introduction: Diet may influence various aspects of human health. In fact, it is well known that diet can favour or not the development of various human pathologies, like diabetes, hypertension, and hypercholesterolaemia. Interestingly, diet has an influence in cancer development too (e.g., this relation has been studied for pancreatic, colonic, gastric, and breast cancers). Between the mechanisms that could explain this relation, there is epigenetic. In fact, thanks to epigenetic reprogramming, certain substances introduced with diet could affect gene expression, especially of those genes involved in cells' proliferation and growth. In recent years, some studies have been published about the role that diet could have on chemotherapy outcome. Especially, various studies have analysed the effects of fasting and ketogenic diet (KD) during chemotherapy. The aim of this study is to
\end{abstract}

summarize scientific evidences about diet and its effects on chemotherapy on humans and to better understand if these approaches deserve to be further investigated and might be suitable and beneficial during cancer treatment. Materials and Methods: We performed an electronic literature search of the PubMed database, using the combination of following terms: "fasting" or "ketogenic" with "chemotherapy," "cancer treatment." We included studies on humans about fasting and KD during chemotherapy, excluding reviews, case series including $<10$ patients, studies conducted on animals or limited to radiotherapy treatment, and studies that were mostly about molecular mechanisms. Results/Discussion In our analysis we included 4 studies ( 1 randomized controlled trial, 1 retrospective study, and 2 prospective pilot studies) about KD and 4 studies ( 1 prospective cohort study, 1 case series report, and 2 randomized trials) about fasting during oncological treatments. Authors suggested an improvement of quality of life (QoL) and fatigue in patients under chemotherapy, especially in the 8 days after chemotherapy treatment. We found that both fasting and KD demonstrated to be tolerable and feasible during oncological treatments.
Francesco Plotti

Department of Obstetrics and Gynecology

Campus Bio Medico, University of Rome, Via A. Del Portillo 200

IT-00128 Rome (Italy)

f.plotti@unicampus.it 
Conversely, data about survival outcomes are still controversial, but it should be underlined that it was not the outcome of these preliminary studies. Conclusions: All comparatives studies have demonstrated that even fasting then KD results in a reduction of collateral effects of adjuvant chemotherapy (due to reduction of drugs toxicity) and a better QoL than in patients that follow no diet. Unfortunately, despite the fact that various laboratory and animal studies confirm advantages from KD and fasting, few data are today disposable on humans: further studies are needed to confirm data exposed in this review.

(c) 2020 S. Karger AG, Basel

\section{Introduction}

Diet may influence various aspects of human health. It is well established that a certain type of diet may favour the development of various illnesses (i.e., diabetes, hypertension, and hypercholesterolaemia) [1]. Particularly, Fontana and Partridge [2] demonstrated that dietary restriction could improve organ function and resistance to stress and, so, health and longevity. It has also been demonstrated that diet may facilitate the development of cancer [3]. In fact, diet may alter the epigenetic programme of our cells and, so, it could modify the expression of some genes, including the ones that are involved in cells' proliferation and growth [4]. For this reason, various studies have been conducted to analyse the connections between the consumption of certain types of food and the development of some cancers, such as pancreatic cancer, colorectal cancer, gastric cancer, breast cancer and so on $[5,6]$.

More recently, some studies have demonstrated the role of diet during chemotherapy, as it could alter the efficacy of therapy. With this regard, Hopkins et al. [7] demonstrated in murine models that hyperglycaemia and the subsequent increase in insulin could compromise chemotherapy effectiveness. Furthermore, in recent years, various studies have also investigated the role of some type of specific diets, such as fasting and ketogenic diet $(\mathrm{KD})[8,9]$ during chemotherapy, trying to clarify whether these approaches may increase chemotherapy's outcome. Most of these studies have been conducted on animal models and there are only some experiences on humans. The aim of this study is to summarize scientific evidences about the role of both fasting and $\mathrm{KD}$ during chemotherapy, with a focus on studies conducted on humans, to better understand if these approaches deserve to be further investigated and might be suitable and beneficial during cancer treatment.

\section{Material and Methods}

\section{Information Sources and Search Strategy}

The search was carried out in December 2018, using MEDLINE as the database. The search strategy was limited to English language articles and there were no restrictions on date of publication. Databases were screened for search terms in either titles or abstracts. Search terms were a combination of "fasting" or "ketogenic" with "chemotherapy," "cancer treatment."

\section{Eligibility Criteria}

Studies

We included either randomized trial (both retrospective and prospective studies) or prospective cohort studies or case series if the number of patients was $>10$. Data from hand searching (meeting proceedings of the Society of Gynaecologic Oncology, the European Society of Medical Oncology, and the American Society of Clinical Oncology) were also included. We excluded reviews and studies conducted on animals or limited to radiotherapy treatment, studies about fasting mimicking diets or that were about molecular mechanisms of fasting and $\mathrm{KD}$, focussing our research on studies on humans receiving chemotherapy. We furthermore excluded not full-text available articles, articles not in English language, articles that were irrelevant for our topic, and case series including $<10$ patients.

\section{Participants}

Eligible patients must be: (1) at least 18 years old; (2) affected by any cancer type; (3) receiving chemotherapy; and (4) receiving dietary adjustments (fasting or KD).

\section{Outcomes}

The outcomes considered were quality of life (QoL), reduction of toxicity from chemotherapy, progression-free survival (PFS), and overall survival (OS).

Study Selection, Data Extraction, and Data Synthesis

Study selection was independently carried out by 2 authors (G.M. and L.F.). Titles and abstracts were screened for eligibility according to inclusion and exclusion criteria. All titles or abstracts considered ineligible were excluded. Differences in judgement during the selection process between the reviewers were discussed in order to find a consensus. Information was collected using an Excel spreadsheet. For each study data about year of publication, study type, cancer type, chemotherapy regimen, sample size, outcome measures, and results were presented. Studies were heterogeneous, and for this reason a meta-analysis was not feasible.

\section{Summary of Findings}

The flow diagram in Figure 1 shows the results of literature search and study selection process. The initial research produced 1,896 works on fasting and $\mathrm{KD}$; applying our inclusion and exclusion criteria (only human patients receiving chemotherapy), we included 4 studies for KD: 1 cohort retrospective study and 3 prospective randomized trials (between them, 1 was a prospective observational study); we included 4 studies for fasting: 1 prospective cohort study, 1 case series report, and 2 prospective randomized trials.
Plotti et al. 
Fig. 1. Study selection.

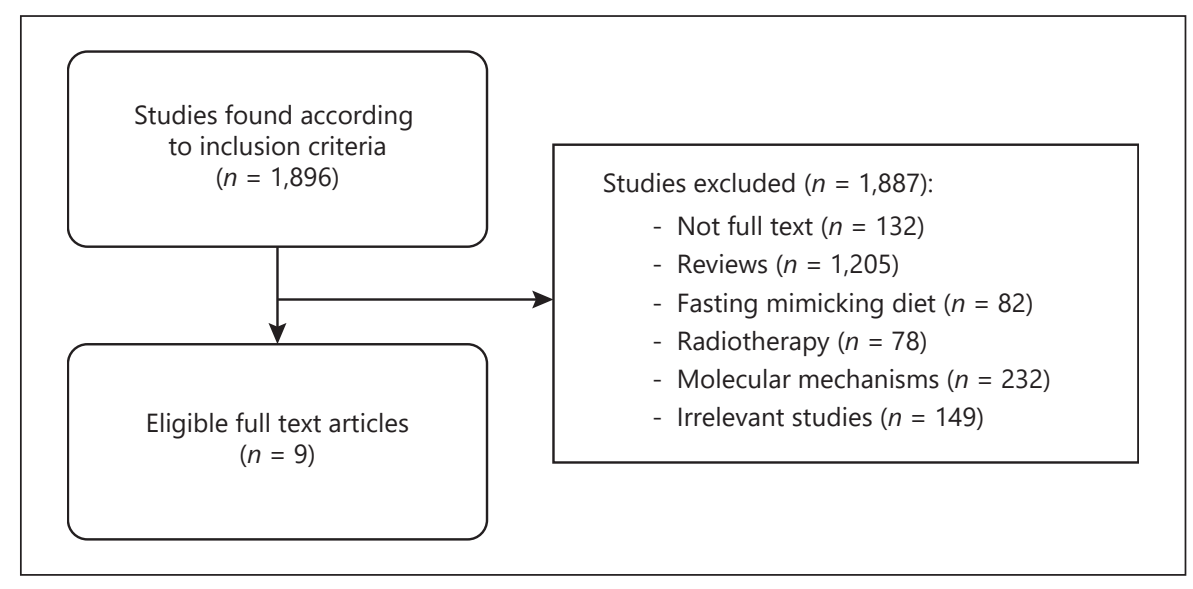

\section{Fasting}

Considering data from laboratory and animals studies, various studies have been to analyse the role of fasting during chemotherapy in humans, with regards of both side effects and outcomes [10]. Bauersfeld et al. [11] conducted a prospective randomized crossover trial, including 50 patients affected by ovarian and breast cancer that underwent various types of chemotherapy (for breast cancer: epirubicin + cyclophosphamide, FU + epirubicin + cyclophosphamide + docetaxel and others; for ovarian cancer: carboplatin or carboplatin + paclitaxel and others). Particularly, they evaluated differences in overall survival, QoL, and fatigue improvement in patients that received normal diet or short-term fasting during chemotherapy. They demonstrated that fasting is safe and it is well tolerated during chemotherapy. Particularly, they demonstrated that fasting might reduce fatigue and improve QoL, especially in the first 8 days after chemotherapy.

To better understand fasting timing during chemotherapy, Dorff et al. [12] conducted a cohort study, including 20 patients undergoing platinum-based chemotherapy for various types of cancer. They compared 24, 48, and $72 \mathrm{~h}$ fasting for the reduction of chemotherapy toxicity. They observed that fasting was safe and feasible for cancer patients and that there was a reduced DNA damage in leucocytes form subjects who fasted for $\geq 48 \mathrm{~h}(p=0.08)$.

Also, de Groot et al. [13] demonstrated a reduced DNA damage in patients undergoing fasting in a prospective randomized trial (in particular a pilot study). They included 13 breast cancer patients that received neoadjuvant chemotherapy with docetaxel, doxorubicin, and cyclophosphamide. They compared normal diet and fasting on side effects, haematological toxicity and DNA damage on mononucleated blood cells. Fasting started $24 \mathrm{~h}$ before chemotherapy and lasted until $24 \mathrm{~h}$ after. Patients could only drink water or tea and coffee without sugar. They demonstrated no significant differences on toxicity induced by chemotherapy in patients undergoing fasting and not, while DNA damages on mononucleated blood cells were reduced in the first $30 \mathrm{~min}$ after therapy administration in fasting patients (considering myeloid cells CD45+ CD3-), suggesting a protective role of fasting in these cells. At the same time, lymphocytes CD45+ CD3+ had the same DNA damage 30 min after therapy administration, but after 7 days, DNA damage remained high only in non-fasting groups, suggesting a role of fasting in promoting DNA recovery after chemotherapy.
Interestingly, Safdie et al. [14] published a case series on 10 patients affected by various types of cancer ( 4 breast, 1 oesophagus, 2 prostate, 1 lung, 1 uterine, and 1 ovarian cancer), undergoing different types of chemotherapy and they focussed on toxicity and side effects after chemotherapy. Fasting period was variable from 1 patient to another, and patients were cases and controls of themselves at the same time. This study showed that fasting may reduce nausea, vomiting, diarrhoea, abdominal pain, and mucositis that were almost absent in the reports from the 10 patients that fasted before or after chemotherapy, while almost 1 of these symptoms was reported by $5 / 6$ patients who received normal feeding. At the same time, the 4 patients that fasted for all the time of chemotherapy reported fewer side effects than patients undergoing the same chemotherapy course without fasting. Furthermore, it was not demonstrated a higher number of side effects in patients who fasted. Studies on fasting are reported in Table 1.

\section{Ketogenic Diet}

KDs are diets that mimic the metabolic state of fasting by inducing a physiological rise in the 2 main circulating ketone bodies, acetoacetate and beta-hydroxybutyrate, above the reference range (typically $0.5 \mathrm{mmol} / \mathrm{L}$ for beta-hydroxybutyrate) [13] These diets are high-fat, adequate protein (about $1.5 \mathrm{~g} / \mathrm{kg}$ body weight), and low-carbohydrate $(<50 \mathrm{~g} /$ day $)$ diets that do not necessarily induce weight loss or micronutrient limitations but mimic fasting to some extent mainly through lowering and stabilizing insulin levels, mildly elevating cortisol levels, and raising fatty acid oxidation; together these adaptions promote hepatic ketogenesis, elevating concentrations of the ketones acetoacetate and D- $b$-hydroxybutyrate [15]. Since the first documented cases of cancer, scientists have been studying the differences between normal cells and tumour cells and how they can resist in different conditions that are prohibitive for human cells [16].

The first experience with $\mathrm{KD}$ during chemotherapy was proposed by Schmidt et al. [17] in 2011, in a prospective observational pilot study on 16 patients affected by advanced cancers with no other possibility of treatment. They wanted to investigate the feasibility of a KD and its influence on the QoL of patients with advanced metastatic tumours.

Sixteen patients with advanced metastatic tumours and no conventional therapeutic options were enrolled and instructed to fol- 

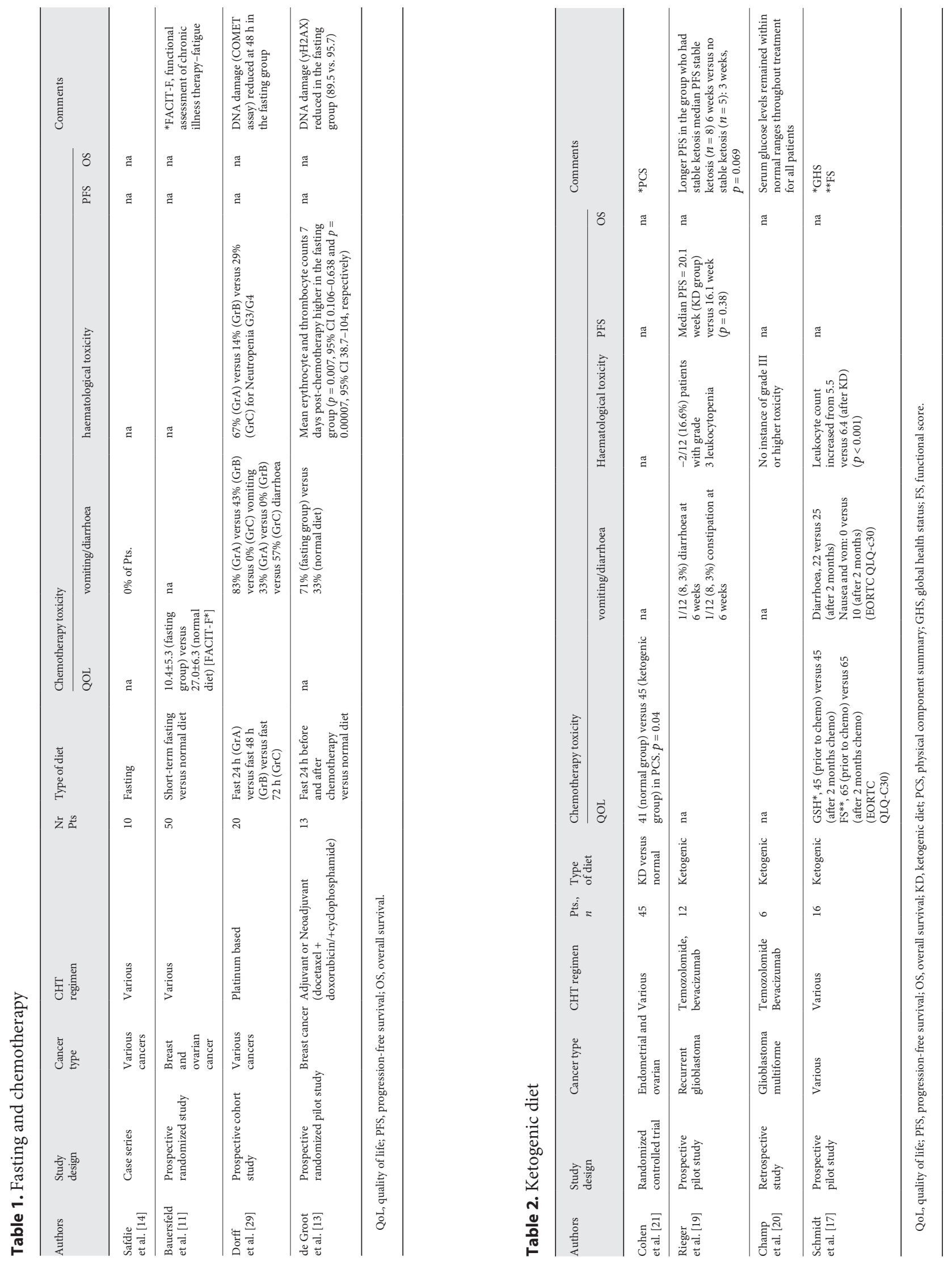
low a $\mathrm{KD}(<70 \mathrm{~g} \mathrm{CHO}$ per day) with normal groceries and were provided with a supply of food additives to mix a protein/fat shake to simplify the 3-month intervention period. QoL modification was evaluated with the EORTC QLQ-C30 questionnaires. The effect of dietary change on metabolism was monitored daily by measuring urinary ketone bodies.

Overall tolerability of KD was as follows: 1 patient did not tolerate the diet and dropped out within 3 days; meanwhile among those who tolerated the diet, 2 patients died early, 1 stopped after 2 weeks due to personal reasons, 1 felt unable to stick to the diet after 4 weeks, 1 stopped after 6 and 2 stopped after 7 and 8 weeks due to progress of the disease, 1 had to discontinue after 6 weeks to resume chemotherapy, and 5 completed the 3-month intervention period. The 5 who competed 3 months of KD and the 1 who resumed chemotherapy after 6 weeks report an improved emotional functioning and less insomnia, while several other parameters of QoL remained stable or worsened, reflecting their very advanced disease. Except for temporary constipation and fatigue, there was no difference in terms of side effects, especially no changes in cholesterol or blood lipids. Accordingly, KD was found to be safe even in a retrospective series of high grade glioma-treated patients, compared with similar patients on an unspecified/standard $\operatorname{diet}[18]$.

In total, 53 patients were analysed. Six underwent a KD during treatment. The diet was well tolerated with no grade III toxicity and 1 episode of grade II fatigue. No episodes of symptomatic hypoglycaemia were registered, and 4 patients were alive after a median follow-up of 14 months. Mean blood glucose of patients on KD were remarkably lower than those on a standard diet (84 vs. $122 \mathrm{mg} / \mathrm{dL}$ ) for those on a KD. Based on this retrospective study, a KD appears safe and well tolerated during the standard treatment of GBM. Authors concluded that dietary restriction of carbohydrates through a KD reduces serum glucose levels significantly, even in conjunction with high dose steroids, with possible effect on response to standard treatment and prognosis.

Ketogenic diet was also proposed on patients with glioblastoma multiforme $[17,19,20]$ and recurrent glioblastoma and it was found to be tolerable and potentially useful in controlling tumour growth. In particular, Rieger et al. [19] published a pilot study with 17 patients affected by recurrent glioblastoma under treatment and on KD. Three patients (15\%) discontinued the diet for poor tolerability. No serious adverse events attributed to the diet were observed. One patient achieved a minor response, and 2 patients had stable disease after 6 weeks.

There were 1 complete response and 5 partial responses in patients treated with bevacizumab, for an overall response rate of $85 \%$, and median PFS on bevacizumab was 20.1 (range, 12-124) weeks, for a PFS at 6 months of $43 \%$. The authors also compared these results with a cohort of 28 patients who were treated with bevacizumab in the same period but who were not on a $\mathrm{KD}$. In these, median PFS was 16.1 weeks (range, 4-90 + weeks; 95\% CI, $15-17$ weeks), showing no difference with the study population on $\mathrm{KD}(p=0.38)$. Interestingly, the effects of a KD alone or in combination with bevacizumab was also explored in an orthotopic U87MG glioblastoma model in nude mice and it was found that KD alone had no effect on median survival but increased that of bevacizumab-treated mice from 52 to 58 days $(p<0.05)$. Finally, Cohen et al. [21] recently conducted a randomized controlled trial with 73 women with ovarian or endometrial cancer $(25 \%$ of these was in concomitant chemotherapy treatment), randomized in 2 arms, 1 including women following $\mathrm{KD}$ and the other including women following ACS (American Cancer Society) diet.

After 12 weeks, there was a significant between-group difference in adjusted physical function scores $(p<0.05)$, and KD participants not receiving chemotherapy reported a significant within-group reduction in fatigue $(p<0.05)$. There were no significant between-group differences in mental function, hunger, or appetite. There was a significant between-group difference in adjusted cravings for starchy foods and fast food fats at 12 weeks $(p<0.05$ for both), with the KD group demonstrating less frequent cravings than the ACS. Also, there was a significant between-group difference in serum beta-hydroxybutyrate concentration at 12 weeks (group means: ACS, $0.250 .04 \mathrm{mmol} / \mathrm{L}$ vs. $\mathrm{KD}, 0.910 .16 \mathrm{mmol} / \mathrm{L}$, $p<0.001)$. Conversely, none differences in terms of QoL was identified between groups. Studies on KD are reported in Table 2.

\section{Discussion}

\section{Fasting}

An increasing body of evidence from experimental research points to beneficial effects of intermittent and periodic fasting in chronic disease: a $20-40 \%$ reduction in calorie intake or dietary restriction protects a wide variety of organisms against oxidative stress and ageing. Similar data were obtained from studies on cancer. In fact, fasting could reduce cancer cells capability to survive and adapt, making them more susceptible to chemotherapy. On the other side, fasting seems to increase the capability of normal cells to resist to chemotherapy treatments and favours normal tissue regeneration. Several mechanisms have been studied to explain these effects [22].

It has been proposed that fasting promotes pronounced changes in metabolic pathways and cellular processes such as stress response, autophagy and decreases insulin-like growth factor 1 that affects other factors such as Akt, Ras, and mTOR to downregulate cell growth and proliferation [7]. Furthermore, ketone bodies produced during fasting can reduce tumour growth and promote cells differentiation through epigenetic mechanisms [23].

Interestingly, as said before, starvation may have different effects on cancer and normal cells. The theory of starvation-dependent differential stress resistance has been proposed by Buschiazzo et al. [24] in 2018. According to this hypothesis, normal cells respond to starvation with a stress response entering in a self-maintenance mode, protecting them from the toxic effects of both radiotherapy and chemotherapy. Otherwise, cancer cells, because of oncogenes mutations, do not have this reaction, hence inhibiting normal stress response pathways. Similar results were shown in a study published by Bianchi et al. [25]. 
Interestingly, various preclinical studies conducted in laboratory on animals or human cancer cells have shown the beneficial effects of fasting on cancer. For example, a greater activity of Sorafenib in human hepatocellular carcinoma cells has been demonstrated in a laboratory study [26]. Also Caffa et al. [27] in 2015 found that fasting could potentiate the effects of chemotherapeutic agents, in particular tyrosine kinase inhibitors, like erlotinib, gefitinib, lapatinib, crizotinib, and regorafenib. In fact, starvation could increase the blockage of cancer cell growth induced by tyrosine kinase inhibitors. Furthermore, fasting could increase the effects of chemotherapy based on cisplatinum too [28].

Considering these preclinical data, in recent years some studies were published about chemotherapy and fasting. Both Safdie et al. [14] and Dorff et al. [29] demonstrated that starvation could decrease chemotherapy toxicity, reducing symptoms like vomiting and nausea and furthermore reducing toxic effects of oncological drugs on cells like leucocytes. Bauersfeld et al. [11], in his prospective randomized crossover trial, found that QoL is less affected by chemotherapy in patients undergoing fasting regimens. On the other side, de Groot et al. [13] did not observe modifications in QoL of patients receiving starvation, but only reduced haematological toxicity. In synthesis, as demonstrated in various animal studies, fasting during chemotherapy could have various beneficial effects on anti-cancer treatment (in terms of QoL and drug toxicity). Unfortunately, only few studies on humans are now disposable and only few of them are prospective studies. Furthermore, they often include a little number of patients affected by various tumours, so that the effects of fasting could be confounding: for example, fasting beneficial effects could be more evident in some tumours than in others, and these heterogeneous studies could hide these effects.

\section{Ketogenic Diet}

Despite advances in developing new chemotherapies and radiation targeting techniques, most of tumours continue to have a poor prognosis. With fasting and $\mathrm{KD}$, we are trying to target fundamental differences between cancer and normal cells; mitochondrial oxidative metabolism with manipulations such as KD could represent a valid implementable strategy for improving outcomes to standard of care radiation and chemotherapy. KDs result in several metabolic changes that may cause stress to cancer cells. Warburg in 1926 described differences in metabolic pathways that lead to production of ATP. He supposed that tumour cells do not use the normal oxidative phosphorylation (that results in $32-36 \mathrm{~mol}$ of ATP) but the aerobic glycolysis that consists in transformation of glucose in pyruvate and then in lactic acid (that results in 2 mol of ATP). This metabolic characteristic is known as the "Warburg effect." Tumour cells' metabolic pathway is definitively disadvantageous than that of the normal human cells, so it results in significantly increased glucose uptake [14]. These principles are widespread in clinical application with the use of PET with the glucose analogue tracer, 18fluorodeoxyglucose [17, 18, 30].

The rationale in providing a fat-rich, low-carbohydrate diet in cancer therapy is to reduce circulating glucose levels and induce ketosis such that cancer cells are starved of energy while normal cells adapt their metabolism to use ketone bodies and survive. Furthermore, by reducing blood glucose, levels of insulin and insulin-like growth factor, which are important drivers of cancer cell proliferation, drop [17]. Fatty acid oxidation occurs primarily in the mitochondria and is dependent upon efficient and well-integrated mitochondrial electron transport chain activity. Cancer cell mitochondria are thought have inefficiencies in mitochondrial electron transport chain activities leading to increased steady-state levels of $\mathrm{O}_{2} / \mathrm{H}_{2} \mathrm{O}_{2}$ and they increase glucose metabolism to generate reducing equivalents to compensate for excess $\mathrm{H}_{2} \mathrm{O}_{2}$. Hypothesis is that limiting consumption of glucose and increasing dependence on oxidation of fatty acids during consumption of a KD would selectively enhance tumour cell versus normal cell sensitivity to radiation and chemotherapy by a mechanism involving oxidative stress. The classical $\mathrm{KD}$ is typically designed to deliver $90 \%$ of calories from fat, $8 \%$ from protein, and $2 \%$ from carbohydrate with a $4: 1$ ratio of fat to protein + carbohydrate. KD was well tolerated by most of the patients included in the studies selected, and adverse events were negligible.

\section{Conclusions}

The role of dietary restriction regimens such as $\mathrm{KD}$ and fasting during cancer treatment with chemotherapy has been investigated via abundant preclinical experiments. However, the conclusions are controversial, and few data are available in human patients. In our analysis we found that both fasting and $\mathrm{KD}$ have demonstrated to be tolerable and feasible during treatment.

Overall, data on fasting are in favour of the safety and feasibility of these approach, with some authors suggesting an improvement even of QoL and fatigue in patients under chemotherapy, especially in the 8 days after che-
Plotti et al. 
motherapy treatment. Conversely, data about survival outcomes are still controversial, but it should be underlined that it was not the outcome of these preliminary studies. In synthesis, all the prospective studies about fasting found that it is able to reduce chemotherapy toxicity and, at the same time, to increase quality of life in patients receiving these approach. At the same time, all studies are concordant about safeness ad feasibility of fasting (according the schemes pro in patients receiving chemotherapy.

Currently, other trials about the role of fasting during chemotherapy are ongoing, with the aim to further investigate the safety and feasibility of short-term fasting prior to administration of chemotherapy or trying to estimate the longest feasible fasting period prior to chemotherapy. Furthermore, even the association of short-term fasting prior to administration of combination chemotherapy with platinum in patients with advanced solid cancers is under evaluation (see Table 1).

On the other hand, KDs seem to have a promising role in the management of oncologic patients, mainly in those affected by tumour of the central nervous system $[31,32]$. Data reported [33] show that oncologic patients in adjuvant treatment might improve their QoL and Code Federal Regulations (adverse event) following a ketogenic diet compared to patients that follow other diets. As for fasting diet, data concerning survival are inconclusive but also very preliminary. However, the role of ketogenic diets on chemotherapy is still unclear, even if it has been supposed that a high-fat diet could facilitate smarter elimination of chemotherapeutical drugs, mainly ones with biliary elimination, reducing adverse events, but data reported are still lacking.
Data about fasting diet and KD on human patients under chemotherapy are still too few in order to draw any definitive conclusion. Nonetheless preliminary results are rather promising and suggest that these approaches other than being feasible and tolerable, might lead to an improvement of oncological outcomes, especially in terms of chemotherapy toxicity and quality of life. Data from further prospective randomized trials are needed to confirm these preliminary data.

\section{Conflict of Interest Statement}

All authors have nothing to disclosure.

\section{Funding Sources}

No funding sources.

\section{Author Contributions}

Substantial contributions to conception and design - Plotti Francesco, Terranova Corrado, Luvero Daniela, Bartolone Martina, Messina Giuseppe, Feole Laura, Cianci Stefano, Scaletta Giuseppe, Marchetti Claudia, Di Donato Violante, Fagotti Anna, Scambia Giovanni, Benedetti Panici Pierluigi, and Angioli Roberto. Drafting and revising the article critically for important intellectual content - Plotti Francesco, Scambia Giovanni, Benedetti Panici Pierluigi, and Angioli Roberto. Final approval of the version to be published - Benedetti Panici Pierluigi, Scambia Giovanni, and Angioli Roberto.

\section{References}

1 Panagiotakos DB, Pitsavos C, Arvaniti F, Stefanadis C. Adherence to the mediterranean food pattern predicts the prevalence of hypertension, hypercholesterolemia, diabetes and obesity, among healthy adults; the accuracy of the medDietScore. Prev Med. 2007; 44(4):335-40.

2 Fontana L, Partridge L. Promoting health and longevity through diet: from model organisms to humans. Cell. 2015;161(1):106-18.

3 Diet and Cancer. World Cancer Research Fund. 2018. Available from: https://www. wcrf.org/dietandcancer.

4 Bishop KS, Ferguson LR. The interaction between epigenetics, nutrition and the development of cancer. Nutrients. 2015;7(2):922-47.

5 Schwingshackl L, Schwedhelm C, Galbete C, Hoffmann G. Adherence to mediterranean diet and risk of cancer: an updated systematic review and meta-analysis. Nutrients. 2017; 9(10): 1063.

6 Stepien M, Chajes V, Romieu I. The role of diet in cancer: the epidemiologic link. Salud Publica Mex. 2016;58(2):261-73.

7 Hopkins BD, Pauli C, Du X, Wang DG, Li X, $\mathrm{Wu}$ D, et al. Suppression of insulin feedback enhances the efficacy of PI3K inhibitors. Nature. 2018;560(7719):499-503.

8 O'Flanagan CH, Smith LA, McDonell SB, Hursting SD. When less may be more: calorie restriction and response to cancer therapy. BMC Med. 2017;15:106.

9 Zahra A, Fath MA, Opat E, Mapuskar KA, Bhatia SK, Ma DC, et al. Consuming a ketogenic diet while receiving radiation and chemotherapy for locally advanced lung cancer and pancreatic cancer: the university of Iowa experience of two phase 1 clinical trials. Radiat Res. 2017;187(6):743-54.

10 Wei M, Brandhorst S, Shelehchi M, Mirzaei $\mathrm{H}$, Cheng CW, Budniak J, et al. Fasting-mimicking diet and markers/risk factors for aging, diabetes, cancer, and cardiovascular disease. Sci Transl Med. 2017;9(377):eaai8700.

11 Bauersfeld SP, Kessler CS, Wischnewsky M, Jaensch A, Steckhan N, Stange R, et al. The effects of short-term fasting on quality of life and tolerance to chemotherapy in patients with breast and ovarian cancer: a randomized crossover pilot study. BMC Cancer. 2018;18(1):476

12 DorffTB, Groshen S, Garcia A, Shah M, TsaoWei D, Pham H, et al. Safety and feasibility of fasting in combination with platinum-based chemotherapy. BMC Cancer. 2016; 16: 360.. 
13 de Groot S, Vreeswijk MP, Welters MJ, Gravesteijn G, Boei JJ, Jochems A, et al. The effects of short-term fasting on tolerance to (neo) adjuvant chemotherapy in HER2-negative breast cancer patients: a randomized pilot study. BMC Cancer. 2015;15:652.

14 Safdie FM, Dorff T, Quinn D, Fontana L, Wei $\mathrm{M}$, Lee $\mathrm{C}$, et al. Fasting and cancer treatment in humans: a case series report. Aging. 2009; 1(12):988-1007.

15 Gatenby RA, Gillies RJ. Why do cancers have high aerobic glycolysis? Nat Rev Cancer. 2004;4(11):891-9.

16 Klement RJ. Beneficial effects of ketogenic diets for cancer patients: a realist review with focus on evidence and confirmation. Med Oncol. 2017;34(8):132.

17 Schmidt M, Pfetzer N, Schwab M, Strauss I, Kämmerer U. Effects of a ketogenic diet on the quality of life in 16 patients with advanced cancer: a pilot trial. Nutr Metab. 2011;8(1):54.

18 Weber DD, Aminazdeh-Gohari S, Kofler B. Ketogenic diet in cancer therapy. Aging. 2018; 10(2):164-5.

19 Rieger J, Bähr O, Maurer GD, Hattingen E, Franz K, Brucker D, et al. ERGO: a pilot study of ketogenic diet in recurrent glioblastoma. Int J Oncol. 2014;44(6):1843-52.

20 Champ CE, Palmer JD, Volek JS, WernerWasik M, Andrews DW, Evans JJ, et al. Targeting metabolism with a ketogenic diet during the treatment of glioblastoma multiforme. J Neurooncol. 2014;117(1):125-31.
21 Cohen CW, Fontaine KR, Arend RC, Soleymani T, Gower BA. Favorable effects of a ketogenic diet on physical function, perceived energy, and food cravings in women with ovarian or endometrial cancer: a randomized, controlled trial. Nutrients. 2018;10(9):1187.

22 Lee C, Raffaghello L, Brandhorst S, Safdie FM, Bianchi G, Martin-Montalvo A, et al. Fasting cycles retard growth of tumors and sensitize a range of cancer cell types to chemotherapy. Sci Transl Med. 2012;4(124):124ra27.

23 Lee C, Longo VD. Fasting vs dietary restriction in cellular protection and cancer treatment: from model organisms to patients. Oncogene. 2011;30(30):3305-16.

24 Buschiazzo A, Cossu V, Bauckneht M, Orengo A, Piccioli P, Emionite L, et al. Effect of starvation on brain glucose metabolism and ${ }^{18} \mathrm{~F}$-2-fluoro-2-deoxyglucose uptake: an experimental in-vivo and ex-vivo study. EJNMMI Res. 2018;8(1):44.

25 Bianchi G, Martella R, Ravera S, Marini C, Capitanio S, Orengo A, et al. Fasting induces anti-Warburg effect that increases respiration but reduces ATP-synthesis to promote apoptosis in colon cancer models. Oncotarget. 2015;6(14):11806-19.

26 Lo Re O, Panebianco C, Porto S, Cervi C, Rappa F, Di Biase S, et al. Fasting inhibits hepatic stellate cells activation and potentiates anticancer activity of sorafenib in hepatocellular cancer cells. J Cell Physiol. 2018;233(2):120212.
27 Caffa I, D’Agostino V, Damonte P, Soncini D, Cea M, Monacelli F, et al. Fasting potentiates the anticancer activity of tyrosine kinase inhibitors by strengthening MAPK signaling inhibition. Oncotarget. 2015;6(14):11820-32.

28 Shi Y, Felley-Bosco E, Marti TM, Orlowski K, Pruschy M, Stahel RA. Starvation-induced activation of $\mathrm{ATM} / \mathrm{Chk} 2 / \mathrm{p} 53$ signaling sensitizes cancer cells to cisplatin. BMC Cancer. 2012;12:571.

29 Dorff TB, Groshen S, Garcia A, Shah M, TsaoWei D, Pham H, et al. Safety and feasibility of fasting in combination with platinum-based chemotherapy. BMC Cancer. 2016;16:360.

30 Hawkins RA, Phelps ME. PET in clinical oncology. Cancer Metastasis Rev. 1988;7(2): $119-42$.

31 Mukherjee P, Augur ZM, Li M, Hill C, Greenwood B, Domin MA, et al. Therapeutic benefit of combining calorie-restricted ketogenic diet and glutamine targeting in late-stage experimental glioblastoma. Commun Biol. 2019;2:200.

32 Abdelwahab MG, Fenton KE, Preul MC, Rho JM, Lynch A, Stafford P, et al. The ketogenic diet is an effective adjuvant to radiation therapy for the treatment of malignant glioma. PLoS One. 2012;7(5):e36197.

33 Strowd RE, Grossman SA. The role of glucose modulation and dietary supplementation in patients with central nervous system tumors. Curr Treat Options Oncol. 2015;16(8):36 